\title{
Partial Discharge Oscillograms and Pulse Distribution in Transformer Oil-Pressboard Insulation
}

\author{
Y.P. Nerkar, S. Senthil Kumar, M.N. Narayanachar and R.S. Nema \\ Department of High Voltage Engineering \\ Indian Institute of Science \\ Bangalore 560 012, INDIA
}

\begin{abstract}
Partial discharges cause degradation of insulation system and a study of their behaviour can give useful information regarding the state of the insulation system. Partial discharge tests are therefore, carried out as quality checks for the insulation system. A model transformer insulation system consisting of pressboard and transformer oil with uniform field electrode arrangement was tested for its behaviour under partial discharges. The system was allowed to age till failure of the sample occurred in the form of a puncture.

Records of partial discharge distributions were acquired along with oscillograms. Oscillograms help in the traceability of the measurement when compared with distributions. A comparative study of the effect of amplifier setting and the pulse repetition rate on the partial discharge distributions and the oscillograms acquired during ageing of the sample has been presented. It is shown that by appropriate threshold on the distribution, distinctive features can be extracted.
\end{abstract}

\section{Introduction}

Oil-pressboard insulation forms a major insulation component in high voltage transformers. Partial discharge (PD) is one of the prime causes for the failure of insulation. Model systems are convenient in studying the $P D$ behaviour of pressboard insulation system. T.R. Blackburn et al [1] have considered a number of examples simulating the many practical cases where PDs develop in an oil wedge formed by insulation interfaces. In the present work, a model insulation consisting of an oil-impregnated pressboard placed between $7 \pi / 12$ uniform field Rogowski electrodes is studied for its PD behaviour. PDs occurred in the wedge shaped oil gap between the pressboard and the electrodes causing degradation of the oil and pressboard sys- tem resulting in failure of the system. Failure occurred due to puncture in the pressboard sample.

\section{Experimental Setup and Procedure}

Straight detection method was used to detect PDs with $50 \mathrm{~Hz}$ ac voltage applied to the test sample. The PD detector was a narrow band instrument whose output was coupled to a multichannel analyser and a digital storage oscilloscope. Records of PD distributions and oscillograms were obtained at regular intervals during ageing of the oil-pressboard system stressed at $13 \mathrm{kV} / \mathrm{mm}$. The distribution records were acquired for a time interval of 10 seconds and oscillograms were obtained for one ac cycle $(20 \mathrm{~ms})$ period.

The experiments consisted of two parts; (a) the calibration experiments and (b) the ageing experiments.

\section{Calibration Experiments}

Calibration experiments were performed to determine the amplifier gain setting (range fixation) to represent the PD distribution in the best possible way. The amplifier system had a maximum output of $8 \mathrm{~V}$ and this required setting of the amplifier gain corresponding to the maximum magnitude of the pulses. Two gain settings corresponding to $200 \mathrm{pC}$ and $1400 \mathrm{pC}$ were used in the calibration experiments.

\section{Amplifier gain setting}

Figure 1 shows an oscillogram and a pulse distribution obtained with amplifier setting of $8 \mathrm{~V}$ corresponding to $200 \mathrm{pC}$. The oscillogram (Figure $1(\mathrm{a})$ ) is an envelop of ten, one cycle records superimposed. When the setting of the amplifier gain was changed to $1400 \mathrm{pC}$ range, the resulting oscillogram and the 

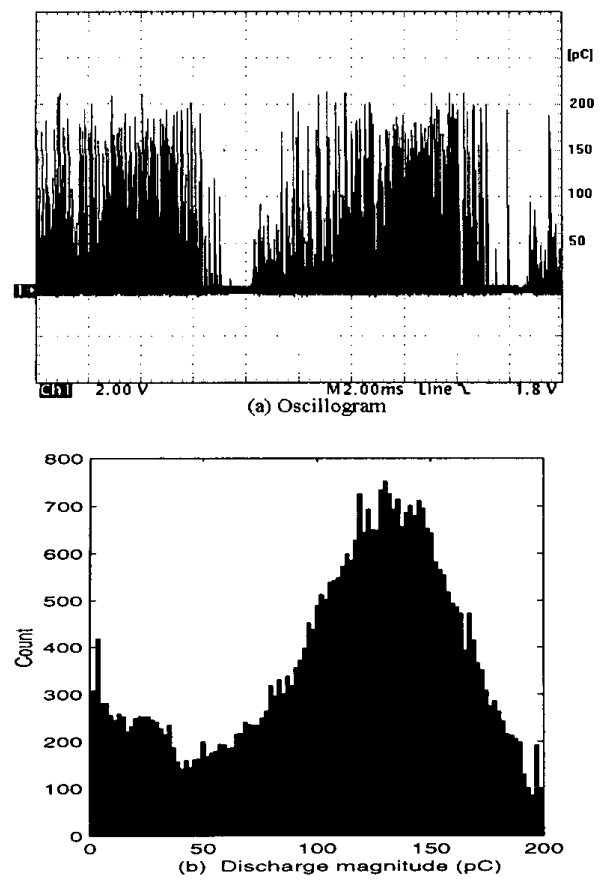

Figure 1: (a) Oscillogram and (b) distribution with $200 \mathrm{pC}$ range

distribution are as shown in Figure 2. The two figures (Figure 1-2) obtained at two different amplifier gain settings depict contrasting features of the PD behaviour.

In Figure 1, the distribution is negatively skewed with very few pulses at the saturation level of $8 \mathrm{~V}$ $(200 \mathrm{pC})$ and many pulses in the mid range $(150 \mathrm{pC})$. This observation is also indicated by the oscillogram (Figure 1(a)). However, the distribution shows less number of lower magnitude pulses in contrast to the observations made from the oscillogram.

In Figure 2, the distribution is positively skewed. The pulse count is reduced and there are many large magnitude pulses $(\geq 200 \mathrm{pC})$ which are not observed as saturated pulses of $8 \mathrm{~V}$ when the gain setting was $200 \mathrm{pC}$ (Figure $1(\mathrm{~b})$ ) and this effect is prominent at phase positions where the number intensity of pulses are high (observation from oscillograms, Figure 1(a) and Figure 2(a)).

\section{Discussion}

Amplifier setting with the range of $200 \mathrm{pC}$ showed the presence of large number of pulses. When observed closely, many of the pulses were found to be overlapping as pulses appeared with a very high repetition rate and within the resolution
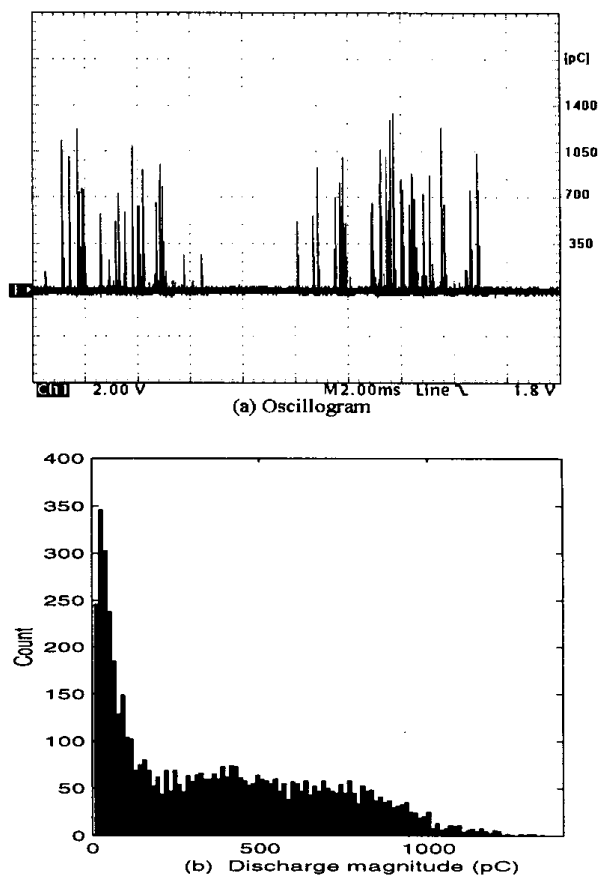

Figure 2: (a) Oscillogram and (b) distribution with $1400 \mathrm{pC}$ range

time $(60 \mu s)$ of the narrow band system. This overlap due to superposition of pulses reduce the pulse magnitudes of subsequent pulses.

That, errors due to superposition of pulses can occur is demonstrated with the help of Figure 3. Figure 3(a) shows the calibrator pulse (CH1), the measuring impedance output (CH2) and the response (CH3) of the narrow band amplifier. Figure 3(b) shows the response of the amplifier when the calibrator pulse frequency is increased and overlapping of the pulses occurs. It can be seen that the magnitude of the second pulse becomes less than the magnitude of the first pulse due to superposition. Therefore, when the repetition rate of the pulse is high, superposition of pulses reduce the magnitudes of the subsequent pulses. This apparently could be the reason for obtaining fewer saturated $(8 \mathrm{~V})$ pulses at the amplifier setting of $200 \mathrm{pC}$ which could result in incorrect representation.

Overlapping of the pulses also reduces the discharge count as many of the overlapping pulses will be measured as a single pulse [2]. The smaller pulses have much higher repetition rate compared to the larger pulses. Therefore, the error is likely to be higher for the lower discharge pulses than that for the larger discharge pulses. Hence, the distribution could have low count for smaller discharge pulses (Figure 1(b)). The error due to limited res- 

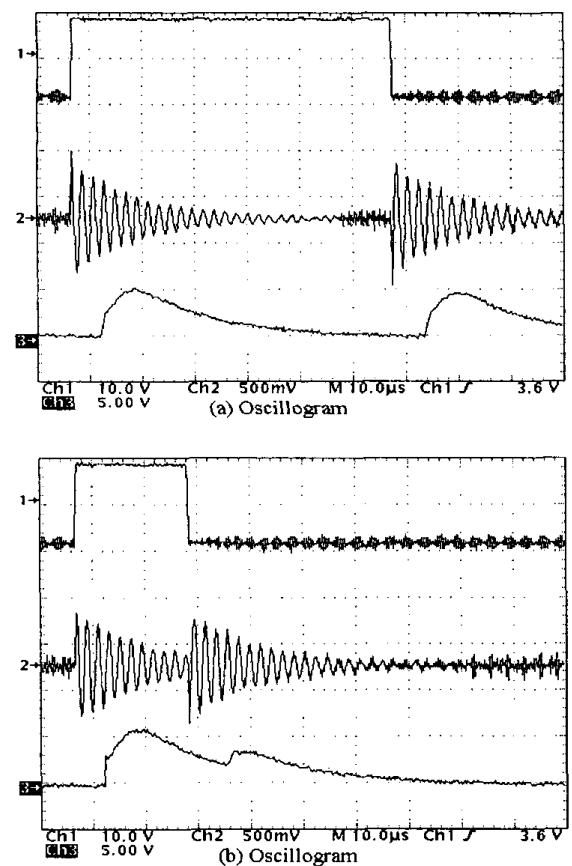

Figure 3: Pulse response (a) without overlap and (b) with overlap error

olution is reduced with the amplifier gain set to 1400 pC. At this gain setting, the smaller magnitude pulses of high repetition rate are automatically eliminated to a large extent due to poor sensitivity of detection.

Hence, one has to be cautious about the amplifier gain setting and the limitation of the detector resolution before attempting interpretation of distribution using shape sensitive statistical and image processing tools.

\section{Ageing Experiments}

The ageing experiments were conducted at a stress of $13 \mathrm{kV} / \mathrm{mm}$ with the amplifier gain setting at the range of $1400 \mathrm{pC}$. The experiment was terminated when the sample failed by puncture. For the 30 number of samples tested, the failure time varied from $37 \mathrm{~min}$ to $194 \mathrm{~min}$. It was observed that, the number of PD pulses, their phase span on the ac cycle and the maximum discharge magnitude increased with ageing. A maximum discharge value of $1000 \mathrm{pC}$ was found to be critical, after which the failure of the sample was imminent.

Typical oscillograms and PD distributions recorded at two instants of time during the course of ageing are given in Figure 4 and Figure 5. Fig-
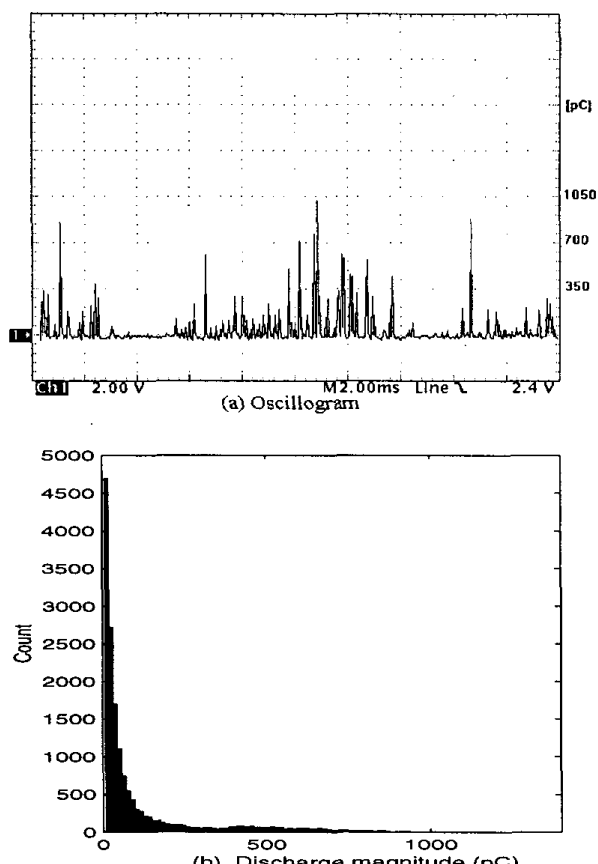

Figure 4: (a) Oscillogram and (b) distribution immediately on application of $13 \mathrm{kV} / \mathrm{mm}$ stress

ure 4(a) and (b) show the oscillogram and the PD distribution obtained immediately after application of voltage stress. Figure 5(a) and (b) show the records obtained just before the failure of the sample.

The oscillograms show that the number intensity of discharge pulses increases with ageing. The distributions show that significant changes occur with larger magnitude pulses (maximum changes in range $500 \mathrm{pC}$ to $1400 \mathrm{pC}$ ). In contrast to the increase in the number intensity as observed from the oscillograms, the count of smaller discharge pulses reduces (Figure 4(b) and Figure 5(b)), just before failure of the sample. This could apparently be due to insufficient resolution of the detector. Therefore, the phenomenon can be safely studied by putting a threshold on the distribution, thus avoiding the ambiguity regarding recording of the smaller magnitude pulses. The effect of putting a threshold on the distribution is shown in Figure 6 which clearly shows the changes occurring in the pulse distribution with ageing. These changes can be analysed with the help of appropriate statistical tools [3].

\section{Conclusions}

With the help of PD oscillograms and their pulse 

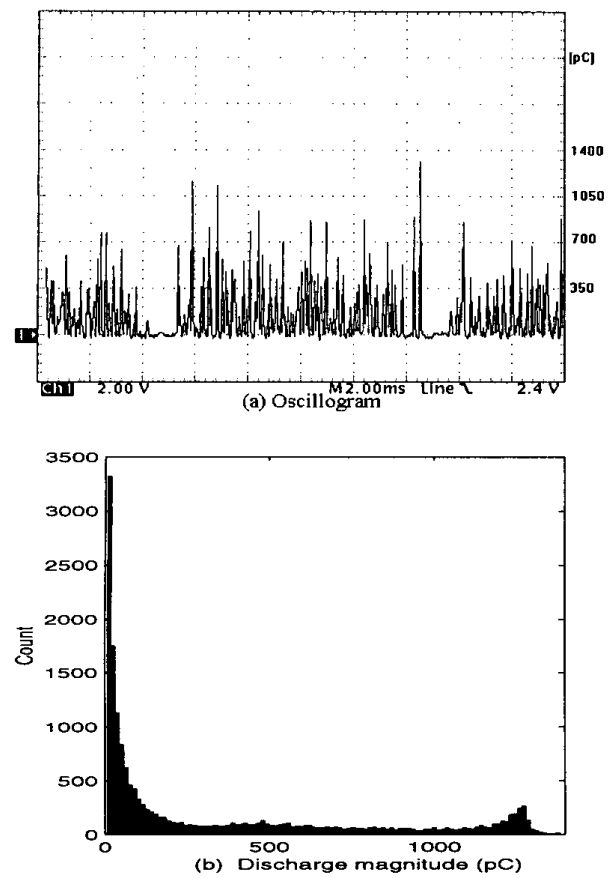

Figure 5: (a) Oscillogram and (b) distribution just before failure

distributions, the importance of amplifier gain setting and the errors that could creep in measurement and analyses are demonstrated. The ageing behaviour of the model insulation system of pressboard-oil sample due to PD is studied. The advantage of setting a suitable threshold and selectively observing the changes in the larger magnitude pulses is also demonstrated.

\section{References}

1. T.R. Blackburn, R.E. James et al, Advanced Techniques for Characterisation of Partial Discharges in Oil-Impregnated and Gas Insulated systems, 15-102, CIGRE, 28 Aug - 3 Sept, 1994 Session.
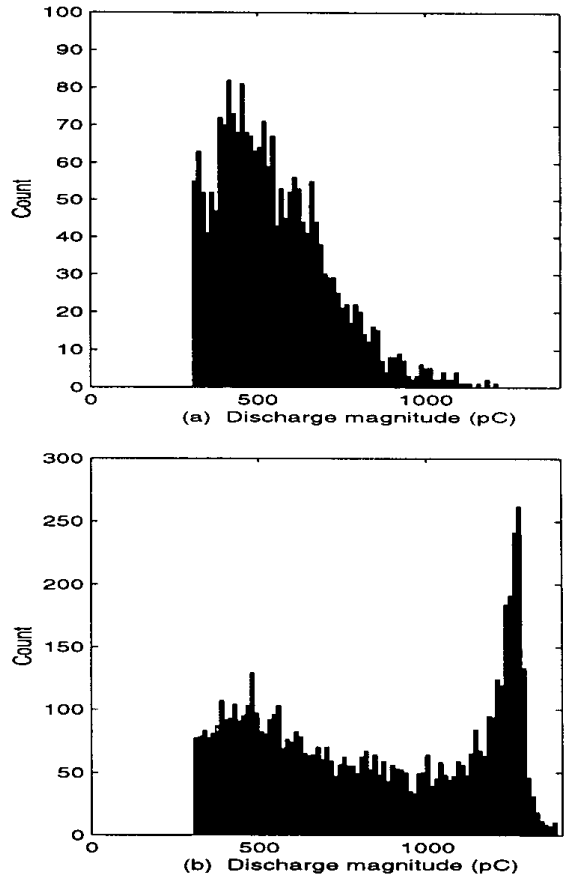

Figure 6: Distributions with threshold (a) immediately after application of $13 \mathrm{kV} / \mathrm{mm}$ stress, (b) just before failure

2. S. Senthil Kumar, M.N. Narayanachar and R.S. Nema, Response of Detectors in the Measurement of Partial Discharges- Proposal for Partial Discharge Detectors with Extended Resolution, Fourth Workshop and Conference on EHV Technology, Bangalore, July 17-18, 1998, pp. 153-157.

3. Y.P. Nerkar, S. Senthil Kumar, M.N. Narayanachar and R.S. Nema, Partial Discharge Oscillograms and Pulse Distribution in Transformer Oil-Pressboard Insulation, Seminar on Transformer Insulation System, Central Power Research Institute, Bangalore, January 28-29, 1999, pp. S VI (1-5). 